Spence refers to the penultimate stage as involving the formation of "activated formic acid", but it is perhaps wise to avoid the nomenclature of organic chemistry, and speak of a "short-lived intermediate" of composition $\left(\mathrm{CH}_{2} \mathrm{O}_{2}\right)$.

One may imagine that the system passes through a series of states defined by symbols $X, X_{1} \ldots X_{x}$ $\ldots X_{(n-2)}, X_{(n-1)}, X_{n}$. To account for the similarity between oxidation and thermal decomposition processes, it seems to be necessary to assume that there is some change which is dominant and common to both, and I suggest that this is to be found in the transition represented by,

$$
X_{(n-2)} \rightarrow X_{(n-1)} .
$$

This is the change involved in the formation of the short-lived intermediates. The probability of reversal of this process is, as Seddon and I suggest, small, and the energy drop considerable. The energy is utilized in a process represented by,

$$
X \rightarrow X_{1},
$$

that is, in increasing the probability of the formation of new primary centres.

The suggestion is somewhat similar to one of those put forward by Semenoff. Qualitatively, it would involve the initial acceleration of the process, which would afterwards slow down. In a considerable number of processes, involving relatively small energy changes, which have been investigated in this laboratory by means of detailed analyses, the form of the $x-t$ graphs is very similar, showing a very sudden transition from rapid to slow reaction generally at or before 50 per cent change. This seems to happen in the case of the oxidation of formaldehyde and in the thermal decomposition of acetaldehyde.

Chemistry Department, University, Bristol.

1 Fletcher, Proc. Roy. Soc., A, 146, 357.

3ravers and others, Proc., Roy. Soc., A, 146, 250, and in the press. 3. Chem. Soc., 649 (1936).

\section{Vitamin P: Flavonols as Vitamins}

VARIous chemical and clinical observations have led to the assumption that ascorbic acid is accompanied in the cell by a substance of similar importance and related activity. In absence of both substances, the symptoms of lack of ascorbic acid (scurvy) prevail and conceal symptoms of the deficiency of the second substance. In the lack of suitable experimental animals or conditions, progress was dependent on spontaneous pathological conditions, caused or influenced by this second factor.

In collaboration with L. Armentano and A. Bensáth, we have found that in certain pathological conditions, characterised by an increased permeability or fragility of the capillary wall, ascorbic acid is ineffective, while the condition can readily be cured by the administration of extracts of Hungarian red pepper ('vitapric') or lemon juice. 'The extracts were effective in cases of decreased resistance of the capillary wall toward whole blood (vascular type of hæmorrhagic purpura) as well as in cases in which the capillary wall showed an increased permeability towards plasma protein only (various septic conditions). The extracts were fractionated. The active substance was found in the end in a fraction consisting of practically pure flavon or flavonol glycoside. $40 \mathrm{mgm}$. of this fraction given daily intravenously to man restored in a fortnight regularly the normal capillary resistance. Spontaneous bleeding ceased, the capillary walls lost their fragility towards pressure differences and no more plasma protein left the vascular system on increased venous pressure.

These results suggest that this great group of vegetable dyes, the flavons or flavonols, also play an important role in animal life, and that the dyes are of vitamin nature. The group is not to be confused with the yellow dye, discovered by one of us and termed 'flaves' (like cytoflave), which dye forms the prosthetic group of Warburg's yellow enzyme and has later been renamed by R. Kuhn 'flavins'. We propose to give the name 'vitamin $P$ ' to the substance responsible for the action on vascular permeability.

This research is sponsored by the Josiah Macy $\mathbf{J}_{1}$. Foundation, Now York.

\section{Medical Clinic and}

ST. RUSZNYÁ.

A. SzENT-GYörgyı.

Biochemical Department,

University, Szeged. May 27.

\section{Role of Adenylic Acid in Vitamin $B_{1}$ Deficiency}

THE work of Peters ${ }^{1}$ and his collaborators has shown that abnormal amounts of lactic and pyruvic acid accumulate in the brain of the polyneuritic pigeon. Further evidence of the Oxford school has demonstrated that the brain tissue of such poly. neuritic pigeons exhibits a lowered oxygen consumption in the presence of either pyruvic or lactic acid. The addition of vitamin $\mathbf{B}_{1}$ in vitro to such tissue restores the oxygen uptake to a large extent. Other workers ${ }^{2}$ have also pointed out the presence of excessive amounts of lactic acid in the tissues of the vitamin $B_{1}$ deficient animal. This emphasis on the 'lactic acid' theory of the vitamin $B_{1}$ syndrome led Drury, Harris and Maudsley ${ }^{3}$ to suggest that the bradycardia found by them in vitamin $B_{1}$ deficient rats was due to the excessive accumulation of lactic acid in the heart. No direct evidence was brought forward to support this theory, although the results of Birch and Harris ${ }^{4}$ showed that the severity of the bradycardia went parallel with the lactic acid level in the blood.

The attention which has been devoted to this theory has obscured the similarity between the effect of adenine nucleotides on the normal heart and the effect of vitamin $\mathbf{B}_{\mathbf{1}}$ deficiency on this organ. In the rat, the bradycardia produced by certain adenine compounds apparently resembles in all respects that produced by avitaminosis $B_{1}$. For example, with moderate avitaminosis or with small doses of adenylic acid, the heart exhibits a simple slowing. In severe avitaminosis when the heart rate is below 350 beats per minute the auricular wave disappears ${ }^{3}$. This disappearance of the auricular wave also occurs when a large enough dose of adenylic acid is injected.

These facts have led us to study the action of adenine nucleotides on the heart of both the normal and vitamin $B_{1}$ deficient animal.

It was found possible to increase further the bradycardia already present, due to vitamin $B_{1}$ deficiency, by the injection of small amounts of adenosine, or either of the adenylic acids from muscle or yeast. Using normal animals, a much smaller effect was 